\title{
Coordinating production quantities and demand forecasts through penalty schemes
}

\author{
MURUVVET CELIKBAS ${ }^{1}$, J. GEORGE SHANTHIKUMAR ${ }^{1,2}$ and JAYASHANKAR M. SWAMINATHAN ${ }^{2}$ \\ ${ }^{1}$ Department of Industrial Engineering and Operations Research and ${ }^{2}$ Walter A. Haas School of Business, University of California at \\ Berkeley, Berkeley CA, 94720, USA \\ E-mail: celikbas@ieor.berkeley.edu
}

Received December 1997 and accepted November 1998

\begin{abstract}
In this paper, we study coordination mechanisms through penalty schemes between manufacturing and marketing departments which enable organizations to match demand forecasts with production quantities. This research was motivated by our interactions with a leading electronics and computer manufacturer. We consider two possible organizational structures - centralized and decentralized. In the decentralized case we model a single period problem where demand is uncertain and the marketing department provides a forecast to manufacturing which in turn produces a quantity based on the forecast and the demand distribution. In the centralized case, marketing and manufacturing jointly decide on the production quantity. Among other results we show that by setting suitable penalties one can generate the same result in a decentralized system as that obtained from a centralized system. We also show that setting the correct penalty for marketing is essential for coordination. Subsequently, we analyze models where the marketing department has the ability to change the distribution of demand based on efforts (through promotion, advertising and personal relationship with customers). An interesting result indicates that it is possible to set penalties so that a coordinated decentralized system outperforms a centralized system when there are no tangible costs to the firm for the efforts expended by the marketing department.
\end{abstract}

\section{Introduction}

Coordination between marketing and manufacturing departments has been considered a challenging issue by many practitioners and researchers [1]. In many cases, these departments within the same firm often have different and conflicting objectives making it a difficult task to coordinate their activities to generate the optimal solution for the firm as a whole. An important activity for any manufacturing firm relates to planning production and inventory for an uncertain demand. In many firms, there is often a large mismatch between the demand forecast and the actual demand realized, which leads to conflicts between the two departments. Game theory is an appropriate tool for analyzing real situations where multiple agents are involved in a decision process and their actions are inter-related [2]. In the past, there has been a limited application of such models in operations and industrial engineering because researchers were mainly interested in analyzing optimization problems involving a single agent. However, in the last few years, researchers in this area have started addressing issues related to coordination between manufacturing and other departments within an organization $[3,4]$ as well as the coordination of operations within a supply chain [5-7] using game theoretic models. In this paper, we develop incentive mechanisms for coordination between the manufacturing and marketing departments within an organization and compare the performance of centralized and decentralized systems under a stochastic setting.

This research was motivated by our interactions with a leading manufacturer in the electronics and computer industry. The firm was operating in a decentralized manner where marketing provided manufacturing with a point forecast of demand for a future period. Since marketing has closer contact with customers one expects them to have a better understanding of the future demand. However, marketing in most cases was providing forecasts which were far above the actual demand realized. The main reason for this was that the marketing department itself was evaluated based on the total revenues generated. As a result, they over-forecasted to ensure that there are no stock outs. The manufacturing department was evaluated mainly based on the inventory costs and was penalized on occasions when the firm experienced large stock outs. In order to make sure that marketing was reporting the correct realized demand at the end of a period, a central site recorded all the demand information. This information was supplied to both departments so that they had a better estimate of future 
demand distribution. The existing performance evaluation mechanism for the two departments and their response (in terms of forecasting above demand or producing below forecast) led to a lack of trust between the two departments which made it even more difficult to coordinate their activities.

Our aim in this paper is to develop and analyze simple models that study coordination issues between marketing and manufacturing, and, compare centralized and decentralized systems. In a centralized system, the production quantity is determined jointly by marketing and manufacturing, taking the costs and revenues of these departments into account while optimizing the total profits of the two departments together. On the other hand, in a decentralized system, marketing and manufacturing make decisions based on their individual objective functions. In such a system, the marketing department is responsible for giving manufacturing a realistic demand forecast and manufacturing produces a quantity based on the demand distribution and the forecast. In our models, we restrict our attention to a single period where there is stochastic demand for one type of item and there are no capacity restrictions at the manufacturing end. We restrict our attention to single period models mainly because of two reasons. Firstly, single period models adequately capture the dynamics for products that have a very short life time and secondly, a multi-period model that captures the cumulative effects of coordination and forecast evolution, makes the comparison of decentralized and centralized systems complicated. First we consider a basic model and three variants where the demand distribution is fixed. In the basic model, we assume that the demand distribution and its realization are known to both marketing and manufacturing. We develop penalty mechanisms for the decentralized case where marketing is penalized for over-forecasting and manufacturing is penalized for under-supply. We show that regardless of demand distribution the decentralized system can perform as well as the centralized system in terms of overall profits if penalties are set appropriately. In addition, we show that under a suitable penalty scheme, the manufacturing department always produces an amount equal to demand forecast given by the marketing department. The three variants of the model that we consider differ in terms of: (1) manufacturing's knowledge of demand distribution; (2) marketing's overforecasting penalty; and (3) manufacturing's knowledge of actual demand realization, respectively. We show that under all these conditions, there exist penalty schemes which enable a decentralized system to perform as well as a centralized system. In addition, our results indicate that in all these cases coordination can be attained by choosing the right penalty for marketing.

We also consider models where we relate the effort levels of the marketing department with the demand distribution under very general assumptions. In a cen- tralized system, these models determine how much effort would be expended (by marketing) and what quantity would be produced (by manufacturing) in order to maximize the expected combined profits of the two departments. In a decentralized system, the marketing department expends efforts to maximize the expected revenues minus the cost associated with its efforts and any penalties imposed by the corporate management, and, manufacturing produces in order to minimize the expected costs incurred. We consider cases where the cost of the effort by marketing does affect corporate profits (tangible cost of effort), and cases it does not (intangible cost of effort). The corporate management's objective is to attain the maximum profit for the whole firm after sales. We show that if all the effort costs are intangible then the expected profits for the firm are higher in the decentralized system as opposed to a centralized system. Typically, in most firms the costs associated with effort levels are a combination of tangible and intangible costs in which case we provide mechanisms by appropriately setting budgets for the marketing department so that they expend an effort level not greater than the optimal effort required to maximize corporate profits.

The rest of the paper is as follows. In the next section we discuss the related literature. We discuss the basic model and three variants in Section 3. Two models where effort levels are incorporated are discussed in Section 4 and we provide our conclusions in Section 5.

\section{Related literature}

Several researchers in the past have addressed the interdependency of marketing and manufacturing. There is an extensive literature which addresses qualitative issues, conflict areas and potential strategies to coordinate the marketing-manufacturing interface [8-11]. Eliashberg and Steinberg [1] provide an insightful review of the different analytical models in the area of the marketingmanufacturing interface. The models examining the marketing-manufacturing interface have two main approaches: (1) integration of the decisions into a single objective; and (2) coordination of successive decisions by individual departments.

In the first approach, decisions are made simultaneously within a single objective. Among those who integrated marketing and manufacturing decisions, Lee and Kim [12] and Kumar and Hadjinicola [13] have reflected the dependency of demand level on price and advertisements and used these two variables in their production planning decisions. Leitch [14] has analyzed a multiperiod case where he incorporated the diminishing effect of advertisements with time. Abad [15] formulated a continuous time, dynamic model to coordinate marketing, production and finance decisions and suggested a solution procedure based on decomposition of the model 
based on the serial nature of the decisions made by marketing, production and finance "subsystems". Similarly, Damon and Schramm [16] formulated a model for production, marketing and finance sectors. Their model considered a discrete time multi-period setup. They coordinated the decisions using non-linear programming but were not able to suggest a solution procedure to obtain a global optimum. They also constructed a decentralized system and showed that although not optimal, the centralized system led to better results.

The second type of approach addresses decentralized decision-making. Freeland [17] has considered the issue of coordinating the decisions and analyzed the effect of information in a decentralized system for a single period under a deterministic setting. He showed that in the absence of perfect information regarding the unit cost per item, marketing ends up deciding on prices and promotions which are non-optimal. Eliashberg and Steinberg [1] provide a simple example where they demonstrate the differences between a centralized and a decentralized system. Their model assumes that price and lot size are the two decision variables and that the demand function is deterministic and downward sloping in price. They compare and contrast a decentralized system to a centralized system.

Porteus and Whang [3] have considered a system with multiple products (each managed by a marketing manager) in a single period setting where demand and capacity are stochastic and can be affected by effort. Efforts expended by marketing and manufacturing managers increase the demand and capacity respectively. Efforts were considered to be inherent to each manager and could not be observed or controlled by others. They developed two incentive schemes for marketing and manufacturing managers which maximized the profits for the owner of the firm. In the first one, the inventory levels were decided by the owner of the firm. In this case, they showed that the system performance in terms of expected return was the same as the case when the effort levels were set by the owner himself. However, they indicate that such a plan could give rise to insufficient capacity. In the second scheme, they allowed marketing to set inventory levels to avoid the complaints due to the insufficient capacity arising in the first plan. They showed that under a suitable incentive scheme the total returns for the firm were the same as in the previous case.

In our models we analyze a single period case in a stochastic demand environment where marketing provides a point forecast for demand to manufacturing. We do not consider any capacity restrictions for manufacturing. We compare a centralized system with a decentralized system and present penalty schemes that enable both systems to perform identically. In our models with efforts, we assume a general stochastic relationship between demand and effort expended by marketing, and, derive penalty mechanisms that enable a firm to coordinate marketing efforts with manufacturing quantities.

\section{Models with fixed demand distribution}

In this section we analyze models where the demand distribution is fixed (in Section 4, we will consider models where marketing can influence the demand for products in a stochastic sense through the effort expended). We first consider a basic model where the demand distribution is assumed to be known to both marketing and manufacturing. In the centralized system, marketing and manufacturing decide together on the quantity to produce in order to maximize the expected profit. The profit depends on the revenues generated, production cost and salvage cost for excess production. In a decentralized system, marketing provides a point estimate of demand to manufacturing and manufacturing produces a quantity based on this forecast. An over-forecasting (forecast in excess of actual demand) penalty is charged to marketing and an under-supply (manufacturing quantity less than the minimum of the actual demand and forecast ) penalty is charged to manufacturing. Manufacturing produces a quantity which minimizes its expected total cost consisting of production cost, salvage cost of excess production and penalty cost for under-supply. We assume that marketing knows manufacturing's objective and provides a point estimate for demand which maximizes the expected revenue minus the penalty for over-forecasting. Since the under-supply penalty for manufacturing is dependent on the demand estimate of marketing their decisions are coupled.

The timing of events is as follows. First, the corporate management sets the under-supply and over-forecasting penalties. The demand distribution is known to marketing and the corporate management unless specified otherwise. Manufacturing is informed about the demand distribution by the corporate management. In all the models, the decisions are made sequentially. The marketing department is the Stackelberg leader and reveals its point estimate for demand to manufacturing. The manufacturing department, the follower, determines the production level. The demand realization follows production and the departments are penalized if necessary.

The notation used in our models is as follows:

$$
\begin{aligned}
p_{u}= & \text { stock out penalty per item for manufacturing; } \\
p_{o}= & \text { overestimate penalty per item for marketing; } \\
p_{s}= & \text { selling price per item; } \\
c= & \text { production cost per item; } \\
s= & \text { salvage cost per item (applied to leftover } \\
& \text { inventory) } \\
d^{*}= & \text { optimal demand forecast given by marketing } \\
& \text { in the decentralized system ; } \\
q_{m}^{*}(d)= & \text { optimal amount produced by manufacturing } \\
& \text { given a demand estimate } d \text { by marketing in } \\
& \text { the decentralized system; } \\
q_{t}= & \text { production level in the centralized system; }
\end{aligned}
$$


$D=$ demand, assumed to be an absolutely continuous random variable;

$f=$ density function of $D$;

$F=$ cumulative distribution function of $D$;

$F^{-1}=$ inverse of the cumulative distribution function $F$;

$E=$ expectation operator with respect to $D$;

$\phi_{m}=$ manufacturing department's objective in the decentralized system;

$\phi_{m r}=$ marketing department's objective in the decentralized system;

$\phi_{t}=$ joint objective in the centralized system.

\subsection{Basic model: common knowledge of demand distribution and actual demand realization}

In this model, both manufacturing and marketing have complete information about demand realization and the demand distribution.

\subsubsection{Decentralized system}

In the decentralized system, marketing and manufacturing are acting so as to optimize their own objectives. Marketing is expected to provide realistic demand estimates to manufacturing. However, if not penalized for over-forecasting, marketing will give the highest possible estimate for demand as the forecast in order to satisfy all the demand and thereby maximize its revenues from sales. Manufacturing aims to minimize the cost of production and salvage, and tries to meet the demand at the same time. We introduce a penalty for marketing of $p_{o}$ per unit of demand that it over-forecasted. So, marketing's objective is given as follows:

$$
\max \left\{\phi_{m r}(d), d \geq 0\right\},
$$

where $d$ is the marketing's demand forecast,

$$
\phi_{m r}(d)=p_{s} E\left[\min \left\{D, q_{m}^{*}(d)\right\}\right]-p_{o} E[\max \{d-D, 0\}],
$$

and $q_{m}^{*}(d)$ is the amount produced by manufacturing in response for marketing's forecast of $d$. Throughout the paper, we will assume that the selling price $p_{s}$ of an item is fixed and it is greater than $c$ (i.e., $p_{s}>c$ ).

Manufacturing is penalized for under-supply. The penalty depends on both the forecast $d$ given by marketing as well as the actual demand $D$. We assume that manufacturing is penalized for under-supply with respect to the minimum of $d$ and $D$. This implies that if the realized demand is greater than the demand forecast, manufacturing is penalized only for the amount by which the production is below the demand forecast. Similarly when demand realized is lower than the forecast estimated by marketing, manufacturing is penalized only for the amount by which the production is below the realized demand because that is the part of the demand that the firm loses. For this to be viable, we of course, assume that manufacturing will get to know the actual demand real- ized (see model variant 3 where this assumption is relaxed). Therefore, manufacturing's objective is given as follows:

$$
\min \left\{\phi_{m}\left(q_{m}\right), q_{m} \geq 0\right\},
$$

where $q_{m}$ is the manufacturing quantity, and

$$
\begin{aligned}
\phi_{m}\left(q_{m}\right)= & p_{u} E\left[\max \left\{\min \{d, D\}-q_{m}, 0\right\}\right] \\
& +c q_{m}+s E\left[\max \left\{q_{m}-D, 0\right\}\right] .
\end{aligned}
$$

Throughout the paper we will assume that the undersupply penalty $p_{u}$ is greater than $c$ (i.e., $p_{u}>c$ ).

Proposition 1. The optimal quantity $q_{m}^{*}(d)$ produced by manufacturing for a demand forecast d made by marketing, is given by

$$
q_{m}^{*}(d)=\min \left\{F^{-1}\left(\frac{p_{u}-c}{p_{u}+s}\right), d\right\} .
$$

Proof. Observe that for a fixed value of $D$, we have, $\max \left\{\min \{d, D\}-q_{m}, 0\right\}, c q_{m}$ and $\max \left\{q_{m}-D, 0\right\}$, all convex in $q_{m}$. Hence from Equation (4) it is easily seen that $\phi_{m}\left(q_{m}\right)$ is convex in $q_{m}$. For $q_{m} \geq d$ we have,

$$
\phi_{m}\left(q_{m}\right)=c q_{m}+s E\left[\max \left\{q_{m}-D, 0\right\}\right],
$$

which is increasing in $q_{m}$. Therefore, $q_{m}^{*}(d)$ that minimizes $\phi_{m}$ should satisfy $q_{m}^{*}(d) \leq d$. For $q_{m} \leq d$, we have

$$
\begin{aligned}
\phi_{m}\left(q_{m}\right)= & p_{u}\left[\left(d-q_{m}\right)(1-F(d))+\int_{q_{m}}^{d}\left(x-q_{m}\right) f(x) \mathrm{d} x\right] \\
& +c q_{m}+s\left[q_{m} F\left(q_{m}\right)-\int_{0}^{q_{m}} x f(x) \mathrm{d} x\right] .
\end{aligned}
$$

Let $\hat{\phi}_{m}\left(q_{m}\right)$ be an extension of Equation (7) defined for all values of $q_{m}$. That is,

$$
\begin{aligned}
\hat{\phi}_{m}\left(q_{m}\right)= & p_{u}\left[\left(d-q_{m}\right)(1-F(d))+\int_{q_{m}}^{d}\left(x-q_{m}\right) f(x) \mathrm{d} x\right] \\
& +c q_{m}+s\left[q_{m} F\left(q_{m}\right)-\int_{0}^{q_{m}} x f(x) \mathrm{d} x\right], q_{m} \geq 0 .
\end{aligned}
$$

The optimal value $\hat{q}_{m}$ that minimizes $\hat{\phi}_{m}\left(q_{m}\right)$ can be found by setting the first derivative of $\hat{\phi}_{m}\left(q_{m}\right)$ to zero. This gives us,

$$
\hat{q}_{m}=F^{-1}\left(\frac{p_{u}-c}{p_{u}+s}\right) .
$$

Hence if, $\hat{q}_{m} \leq d$, then $q_{m}=\hat{q}_{m}$ will also minimize $\phi_{m}\left(q_{m}\right)$. Otherwise $q_{m}=d$ will minimize $\phi_{m}\left(q_{m}\right)$. Therefore we see that

$$
q_{m}^{*}(d)=\min \left\{F^{-1}\left(\frac{p_{u}-c}{p_{u}+s}\right), d\right\} .
$$


Remark 1. Note that the above solution is intuitive because it is never optimal for manufacturing to produce more than the demand estimate specified by marketing because it will not be penalized for the amount beyond the forecasted demand even if the realization happens to be higher.

Theorem 1. Under the above penalty scheme (when marketing is penalized for over-forecasting and manufacturing for under-supply based on the minimum of forecast and actual demand),

(i) $d^{*}=\min \left\{F^{-1}\left(\frac{p_{s}}{p_{s}+p_{o}}\right), F^{-1}\left(\frac{p_{u}-c}{p_{u}+s}\right)\right\}$, and

(ii) $q_{m}^{*}\left(d^{*}\right)=d^{*}$.

Proof. In Proposition 1, we have seen that

$$
q_{m}^{*}(d)=\min \left\{F^{-1}\left(\frac{p_{u}-c}{p_{u}+s}\right), d\right\} .
$$

As it is the minimum of a constant and a linear function in $d$, it is concave in $d$. Then for a fixed value of $D$, we have, $\min \left\{D, q_{m}^{*}(d)\right\}$ concave in $d$ and $\max \{d-D, 0\}$ convex in $d$. Therefore from Equation (2), it easily follows that $\phi_{m r}(d)$ is concave in $d$. For $d \geq$ $F^{-1}\left(\left(p_{u}-c\right) /\left(p_{u}+s\right)\right)$, we have from Equation (5), $q_{m}^{*}(d)=F^{-1}\left(\left(p_{u}-c\right) /\left(p_{u}+s\right)\right)$ and hence from Equation (2) we get

$$
\begin{aligned}
\phi_{m r}(d)= & p_{s} E\left[\min \left\{D, F^{-1}\left(\frac{p_{u}-c}{p_{u}+s}\right)\right\}\right] \\
& -p_{o} E[\max \{d-D, 0\}],
\end{aligned}
$$

which is decreasing in $d$. Hence $d=d^{*}$ that maximizes $\phi_{m r}(d)$ should satisfy $d^{*} \leq F^{-1}\left(\left(p_{u}-c\right) /\left(p_{u}+s\right)\right)$. For $d \leq F^{-1}\left(\left(p_{u}-c\right) /\left(p_{u}+s\right)\right)$, we have $q_{m}^{*}(d)=d$ and hence,

$$
\phi_{m r}(d)=p_{s} E[\min \{D, d\}]-p_{o} E[\max \{d-D, 0\}] .
$$

Let

$$
\begin{aligned}
\hat{\phi}_{m r}(d)= & p_{s}\left[\int_{0}^{d} x f(x) \mathrm{d} x+(d)(1-F(d))\right] \\
& -p_{o}\left[(d) F(d)-\int_{0}^{d} x f(x) \mathrm{d} x\right], d \geq 0 .
\end{aligned}
$$

Note that $\phi_{m r}(d)=\hat{\phi}_{m r}(d)$ for $d \leq F^{-1} \quad\left(\left(p_{u}-c\right) /\right.$ $\left.\left(p_{u}+s\right)\right)$. The optimal value $d=\hat{d}$ that maximizes $\hat{\phi}_{m r}(d)$ can be obtained by setting the derivative of $\hat{\phi}_{m r}(d)$ to zero. Then we get

$$
\hat{d}=F^{-1}\left(\frac{p_{s}}{p_{s}+p_{o}}\right) .
$$

Therefore, if $\hat{d} \leq F^{-1}\left(\left(p_{u}-c\right) /\left(p_{u}+s\right)\right)$, we see that $d=\hat{d}$ will also maximize $\phi_{m r}(d)$. Otherwise, $d=$ $F^{-1}\left(\left(p_{u}-c\right) /\left(p_{u}+s\right)\right)$ will maximize $\phi_{m r}(d)$. Therefore $d^{*}=\min \left\{F^{-1}\left(\left(p_{s}\right) /\left(p_{s}+p_{o}\right)\right), \quad F^{-1}\left(\left(p_{u}-c\right) /\left(p_{u}+s\right)\right)\right\}$. Since $d^{*} \leq F^{-1}\left(\left(p_{u}-c\right) /\left(p_{u}+s\right)\right)$, from Proposition 1 we see that $q_{m}^{*}\left(d^{*}\right)=d^{*}$.

Remark 2. The above result is intuitive because marketing is aware that the production quantity is bounded by a constant, namely $F^{-1}\left(\left(p_{u}-c\right) /\left(p_{u}+s\right)\right)$, which limits the revenues from sales. By giving demand estimates greater than this amount, marketing will only be subject to higher penalty for over estimation without any increase in revenues, which is obviously not appealing to marketing.

\subsubsection{Centralized system}

In a centralized system, marketing and manufacturing join together to determine the amount to be produced with the objective of maximizing their combined profits. The objective for corporate profits is given as follows.

$$
\max \left\{\phi_{t}\left(q_{t}\right), q_{t} \geq 0\right\},
$$

where $q_{t}$ is the production quantity, and

$$
\phi_{t}\left(q_{t}\right)=p_{s} E\left[\min \left\{q_{t}, D\right\}\right]-s E\left[\max \left\{q_{t}-D, 0\right\}\right]-c q_{t} .
$$

The above formulation is similar to a news-vendor problem and the results can be summarized as follows.

Proposition 2. $\phi_{t}$ is concave in $q_{t}$ and the optimal quantity $q_{t}^{*}$ that maximizes $\phi_{t}\left(q_{t}\right)$ is given by,

$$
q_{t}^{*}=F^{-1}\left(\frac{p_{s}-c}{p_{s}+s}\right) .
$$

Now we develop a penalty scheme so that the decentralized system performs as well as the centralized system in terms of overall corporate profit. Note that $p_{o}$ and $p_{u}$ are internal penalties that are assigned to marketing and manufacturing but they do not affect the actual corporate profit in any way. In order to obtain a similar performance from both centralized and decentralized systems, the penalty scheme needs to make certain that the optimal quantities produced in both cases are the same.

Theorem 2. If (i) $p_{u}=p_{s}$ and (ii) $p_{o}=p_{s}(c+s) /\left(p_{s}-c\right)$, then the optimal quantities produced and sold in both centralized and decentralized systems are identical. Specifically $d^{*}=q_{m}^{*}\left(d^{*}\right)=q_{t}^{*}$.

Proof. From Theorem 1, we know that $d^{*}=q_{m}^{*}\left(d^{*}\right)=$ $\min \left\{F^{-1}\left(\left(p_{s}\right) /\left(p_{s}+p_{o}\right)\right), F^{-1}\left(\left(p_{u}-c\right) /\left(p_{u}+s\right)\right)\right\}$. Let us consider two cases: (1) $d^{*}=q_{m}^{*}\left(d^{*}\right)=F^{-1}\left(\left(p_{s}\right) /\left(p_{s}+p_{o}\right)\right)$ and (2) $d^{*}=q_{m}^{*}\left(d^{*}\right)=F^{-1}\left(\left(p_{u}-c\right) /\left(p_{u}+s\right)\right)$. We know from Proposition 2 that $q_{t}^{*}=F^{-1}\left(\left(p_{s}-c\right) /\left(p_{s}+s\right)\right)$. In order for $q_{m}^{*}\left(d^{*}\right)=q_{t}^{*}$ we need the following conditions. 
Case (1):

$$
\begin{gathered}
F^{-1}\left(\frac{p_{s}-c}{p_{s}+s}\right)=F^{-1}\left(\frac{p_{s}}{p_{s}+p_{o}}\right), \\
\Leftrightarrow \frac{p_{s}}{p_{s}+p_{o}}=\frac{p_{s}-c}{p_{s}+s} \\
\Leftrightarrow p_{o}=\frac{p_{s}(s+c)}{p_{s}-c} .
\end{gathered}
$$

Case (2):

$$
\begin{gathered}
F^{-1}\left(\frac{p_{u}-c}{p_{u}+s}\right)=F^{-1}\left(\frac{p_{s}-c}{p_{s}+s}\right), \\
\Leftrightarrow \frac{p_{u}-c}{p_{u}+s}=\frac{p_{s}-c}{p_{s}+s}, \\
\Leftrightarrow p_{u}=p_{s} .
\end{gathered}
$$

Thus, if $p_{u}=p_{s}$ and $p_{o}=p_{s}(c+s) /\left(p_{s}-c\right)$, then we have

$$
\begin{aligned}
F^{-1}\left(\frac{p_{s}}{p_{s}+p_{o}}\right) & =F^{-1}\left(\frac{p_{u}-c}{p_{u}+s}\right)=F^{-1}\left(\frac{p_{s}-c}{p_{s}+s}\right), \\
& \Rightarrow d^{*}=q_{m}^{*}\left(d^{*}\right)=q_{t}^{*} .
\end{aligned}
$$

That is, the optimal quantities produced and sold in both centralized and decentralized systems are identical.

Remark 3. It is important to note that the penalty scheme we have here to coordinate the marketing forecast $d^{*}$, manufacturing quantity $q_{m}^{*}\left(d^{*}\right)$ and the globally optimal production quantity $q_{t}^{*}$, is independent of the demand distribution. All we require (at this stage) is that the demand distribution be known to both marketing and manufacturing. As we will see soon (in model variant 1), this requirement can be relaxed. But, as for the coordinating penalty scheme we have, it appears that: (i) the over-estimate penalty be set such that $p_{o}=p_{s}(c+s) /$ $\left(p_{s}-c\right)$; and (ii) the under-supply penalty be set such that $p_{u}=p_{s}$. Suppose we set $p_{u}>p_{s}$. Then for $p_{o}=p_{s}(c+s) /$ $\left(p_{s}-c\right)$, we have

$$
\frac{p_{u}-c}{p_{u}+s}>\frac{p_{s}-c}{p_{s}+s}=\frac{p_{s}}{p_{s}+p_{o}} .
$$

Hence

$$
\begin{aligned}
d^{*} & =\min \left\{F^{-1}\left(\frac{p_{s}}{p_{s}+p_{o}}\right), F^{-1}\left(\frac{p_{u}-c}{p_{u}+s}\right)\right\} \\
& =F^{-1}\left(\frac{p_{s}}{p_{s}+p_{o}}\right)=F^{-1}\left(\frac{p_{s}-c}{p_{s}+s}\right)=q_{t}^{*} .
\end{aligned}
$$

and

$$
d^{*}=q_{m}^{*}\left(d^{*}\right)=q_{t}^{*} .
$$

On the other hand, suppose we set $p_{o}<p_{s}(c+s) /$ $\left(p_{s}-c\right)$. Then for $p_{u}=p_{s}$, we have

$$
\frac{p_{s}}{p_{s}+p_{o}}>\frac{p_{s}-c}{p_{s}+s}=\frac{p_{u}-c}{p_{u}+s} .
$$

Then as before we have, $d^{*}=q_{m}^{*}\left(d^{*}\right)=q_{t}^{*}$. In the first case, manufacturing is over-penalized and in the second case marketing is under-penalized. It is interesting to note that either by over-penalizing manufacturing or by underpenalizing marketing we can still coordinate the system such that the centralized solution is achieved. That is, both marketing and manufacturing departments optimize the joint profits while they optimize their own objectives. In this way, the decentralized system performs as well as the centralized system. Thus we have the following corollary to Theorem 2 .

Corollary 1. If (i) $p_{u}=p_{s}$ and $p_{o} \leq p_{s}(c+s) /\left(p_{s}-c\right)$ or (ii) $p_{u} \geq p_{s}$ and $p_{o}=p_{s}(c+s) /\left(p_{s}-c\right)$ then the optimal quantities produced and sold in both centralized and decentralized systems are identical. Specifically $d^{*}=$ $q_{m}^{*}\left(d^{*}\right)=q_{t}^{*}$.

Remark 4. It should be noted that if manufacturing is over-penalized and marketing is under-penalized, the decentralized system will result in over-production (that is, $\left.q_{m}^{*}\left(d^{*}\right)>q_{t}^{*}\right)$. In all other cases (other than the three cases we considered where $\left.q_{m}^{*}\left(d^{*}\right)=q_{t}^{*}\right)$, under-production will result (see Table 1 below).

\subsection{Model variant 1: demand distribution unknown to the corporate management}

Since in many firms marketing is the department that deals directly with the customers, it may be argued that at an internal level, corporate management may not know the actual demand distribution. In this section we show that even in this case, it is possible to develop a penalty scheme by which we can have separate (that is, decentralized) decision making and still end up with a coordinated system that will attain the same solution as a centralized system.

Let us assume that the corporate estimate of the demand distribution is $\hat{F}$ (possibly different from the true demand distribution $F$ ). So manufacturing will use $\hat{F}$ to decide its production quantities and thus, in this case it can be shown that (see Proposition 1)

$$
q_{m}^{*}(d)=\min \left\{\hat{F}^{-1}\left(\frac{p_{u}-c}{p_{u}+s}\right), d\right\} .
$$

Then it can be established that (see Theorem 2),

Table 1. Penalty schemes and coordination effects

\begin{tabular}{llll}
\hline & $p_{o}<\frac{p_{s}(c+s)}{p_{s}-c}$ & $p_{o}=\frac{p_{s}(c+s)}{p_{s}-c}$ & $p_{o}>\frac{p_{s}(c+s)}{p_{s}-c}$ \\
\hline$p_{u}<p_{s}$ & $q_{m}^{*}\left(d^{*}\right)<q_{t}^{*}$ & $q_{m}^{*}\left(d^{*}\right)<q_{t}^{*}$ & $q_{m}^{*}\left(d^{*}\right)<q_{t}^{*}$ \\
$p_{u}=p_{s}$ & $q_{m}^{*}\left(d^{*}\right)=q_{t}^{*}$ & $q_{m}^{*}\left(d^{*}\right)=q_{t}^{*}$ & $q_{m}^{*}\left(d^{*}\right)<q_{t}^{*}$ \\
$p_{u}>p_{s}$ & $q_{m}^{*}\left(d^{*}\right)>q_{t}^{*}$ & $q_{m}^{*}\left(d^{*}\right)=q_{t}^{*}$ & $q_{m}^{*}\left(d^{*}\right)<q_{t}^{*}$ \\
\hline
\end{tabular}




$$
d^{*}=q_{m}^{*}\left(d^{*}\right)=\min \left\{F^{-1}\left(\frac{p_{s}}{p_{s}+p_{o}}\right), \hat{F}^{-1}\left(\frac{p_{u}-c}{p_{u}+s}\right)\right\} .
$$

Theorem 3. If

(i) $p_{u}=\left(c+s \hat{F}\left(F^{-1}\left(\frac{p_{s}-c}{p_{s}+s}\right)\right)\right) /\left(1-\hat{F}\left(F^{-1}\left(\frac{p_{s}-c}{p_{s}+s}\right)\right)\right)$,

and $p_{o} \leq p_{s}(c+s) /\left(p_{s}-c\right)$,

or

(ii) $p_{u} \geq\left(c+s \hat{F}\left(F^{-1}\left(\frac{p_{s}-c}{p_{s}+s}\right)\right)\right) /\left(1-\hat{F}\left(F^{-1}\left(\frac{p_{s}-c}{p_{s}+s}\right)\right)\right)$,

and $p_{o}=p_{s}(c+s) /\left(p_{s}-c\right)$, then the optimal quantities produced and sold in both centralized and decentralized systems are identical. Specifically $d^{*}=q_{m}^{*}\left(d^{*}\right)=q_{t}^{*}$.

Proof. Follows from an argument similar to that which led to Corollary 1.

Remark 5. To implement this penalty scheme, someone at the corporate level needs to have knowledge of the actual demand distribution $F$ and the penalty schemes will change depending on the distributions. Thus, this scheme appears to be not practical. We may, however, simplify the above scheme into one that is more practical. For this we need to ensure that the manufacturing department uses an $\hat{F}$ that is not completely arbitrary in comparison to $F$. First we need to ensure that

$$
\inf \{x: F(x)=1, x \geq 0\} \leq \inf \{x: \hat{F}(x)=1, x \geq 0\} .
$$

That is, manufacturing does not under-estimate the largest possible demand value. In that case $\hat{F}\left(F^{-1}\left(\left(p_{s}-c\right) /\right.\right.$ $\left.\left.\left(p_{s}+s\right)\right)\right)<1$ and hence

$$
\left(c+s \hat{F}\left(F^{-1}\left(\frac{p_{s}-c}{p_{s}+s}\right)\right)\right) /\left(1-\hat{F}\left(F^{-1}\left(\frac{p_{s}-c}{p_{s}+s}\right)\right)\right)<\infty
$$

We may then choose a very large $p_{u}$ and satisfy

$p_{u} \geq\left(c+s \hat{F}\left(F^{-1}\left(\frac{p_{s}-c}{p_{s}+s}\right)\right)\right) /\left(1-\hat{F}\left(F^{-1}\left(\frac{p_{s}-c}{p_{s}+s}\right)\right)\right)$.

Suppose we ensure that

$$
\hat{F}(x) \leq F(x), \quad x \geq 0 .
$$

That is, the manufacturing uses a distribution that is stochastically larger than the true demand distribution (that is what the marketing department would like manufacturing to do in a real situation anyway!). Note that stochastic dominance implies (19). Then, it is easily verified that

$$
\left(c+s \hat{F}\left(F^{-1}\left(\frac{p_{s}-c}{p_{s}+s}\right)\right)\right) /\left(1-\hat{F}\left(F^{-1}\left(\frac{p_{s}-c}{p_{s}+s}\right)\right)\right) \leq p_{s} .
$$

Then $p_{u} \geq p_{s}$ will be sufficient to satisfy

$$
p_{u} \geq\left(c+s \hat{F}\left(F^{-1}\left(\frac{p_{s}-c}{p_{s}+s}\right)\right)\right) /\left(1-\hat{F}\left(F^{-1}\left(\frac{p_{s}-c}{p_{s}+s}\right)\right)\right) .
$$

Theorem 4. Suppose manufacturing uses a demand distribution $\hat{F}$ that is stochastically larger than $F$ (that is $\hat{F}(x) \leq F(x), x \geq 0)$. If $p_{u} \geq p_{s}$ and $p_{o}=p_{s}(c+s) /$ $\left(p_{s}-c\right)$, then the optimal quantities produced and sold in both centralized and decentralized systems are identical. Specifically $d^{*}=q_{m}^{*}\left(d^{*}\right)=q_{t}^{*}$.

Remark 6. In this model variant, we can coordinate marketing and manufacturing to perform in a globally optimal way by providing the right penalty for marketing, and, by providing a large enough penalty and a stochastically larger demand distribution for manufacturing. Hence a proper control over marketing is essential in this (more realistic) case, since the coordination is entirely carried out through marketing (that is we need to directly control them to choose the right forecast).

Remark 7. One might argue that the manufacturing department may distrust the information from the corporate management in the long run if it realizes that they are giving a stochastically larger distribution. However, the corporate management has better information about the demand than manufacturing and it will be able to update the demand distribution and provide more accurate information over time. In the long run, the demand distribution estimate by the corporate management will approach to the true demand distribution and the model variant will therefore approach to the basic model.

\subsection{Model variant 2: modified over-estimate penalty for marketing}

In some situations, the marketing department may argue that it should be penalized for the overestimated amount if that leads manufacturing to produce more than the realized demand. The argument would be that if the produced amount cannot satisfy the realized demand in any case, why should marketing be penalized for overestimating since manufacturing never produced that much. In this section we consider a model where marketing is penalized for over-forecasting only if manufacturing produced greater than the realized demand. Marketing's penalty is given by $p_{o} \max \{d-D, 0\}$ $\times I\{D \leq q\}$ where $I\{D \leq q\}$ is a $0-1$ indicator function and $q$ is the quantity produced by manufacturing. Here 
marketing is penalized only if $D \leq q$. In this case marketing's objective is as follows:

$$
\max \left\{\phi_{m r}^{(2)}(d), d \geq 0\right\},
$$

where

$$
\begin{aligned}
\phi_{m r}^{(2)}(d)= & p_{s} E\left[\min \left\{D, q_{m}^{*}(d)\right\}\right] \\
& -p_{o} E\left[\max \{d-D, 0\} I\left\{D \leq q_{m}^{*}(d)\right\}\right] .
\end{aligned}
$$

\section{Proposition 3.}

(1) Manufacturing's production level $q_{m}^{*}\left(d^{*}\right)$ will always match with the optimal demand forecast $d^{*}$ from the marketing department.

(2) If (a) $p_{u} \geq p_{s}$ and $p_{o}=p_{s}(c+s) /\left(p_{s}-c\right)$, or (b) $p_{u}=p_{s}$ and $p_{o} \leq p_{s}(c+s) /\left(p_{s}-c\right)$, then manufacturing's production level $q_{m}^{*}\left(d^{*}\right)$ will always match with the globally optimal production quantity $q_{t}^{*}$.

Proof. (1) Manufacturing's objective here is the same as that for the basic model. Therefore, from Proposition 1 we have $q_{m}^{*}(d)=\min \left\{F^{-1}\left(\left(\left(p_{u}-c\right) /\left(p_{u}+s\right)\right), d\right)\right\}$. For $d \geq F^{-1}\left(\left(p_{u}-c\right) /\left(p_{u}+s\right)\right)$ we have $q_{m}^{*}(d)=F^{-1}\left(\left(p_{u}-c\right) /\right.$ $\left.\left(p_{u}+s\right)\right)$. Then, for $d \geq F^{-1}\left(\left(p_{u}-c\right) /\left(p_{u}+s\right)\right)$,

$$
\begin{aligned}
\phi_{m r}^{(2)}(d)= & p_{s} E\left[\min \left\{D, F^{-1}\left(\frac{p_{u}-c}{p_{u}+s}\right)\right\}\right] \\
& -p_{o} E\left[\max \{d-D, 0\} I\left\{D \leq F^{-1}\left(\frac{p_{u}-c}{p_{u}+s}\right)\right\}\right],
\end{aligned}
$$

is clearly decreasing in $d$. The optimal $d^{*}$ that minimizes $\phi_{m r}^{(2)}$ should satisfy $d^{*} \leq F^{-1}\left(\left(p_{u}-c\right) /\left(p_{u}+s\right)\right)$. But for $d \leq F^{-1}\left(\left(p_{u}-c\right) /\left(p_{u}+s\right)\right)$, we have $q_{m}^{*}(d)=d$. This proves (1).

(2) For $q_{m}^{*}(d)=d$, the over-estimation penalty for marketing becomes $p_{o} \max \{d-D, 0\} I\{D \leq d\}=$ $p_{o} \max \{d-D, 0\}$ which is the same as the over-estimation penalty for the basic model. Then (2) follows from Corollary 1.

Remark 8. In this model variant, we can coordinate marketing and manufacturing to perform similar to a centralized case either by providing the right penalty for marketing or by providing the right penalty for manufacturing.

\subsection{Model variant 3: actual demand realization unknown to manufacturing}

In this section, we consider a variant of the basic model where marketing is the only department that experiences the real demand. This is typically the case in firms that do not have fully integrated information systems. As customers arrive one at a time, creating the demand for items, marketing forwards the request for items to be delivered by manufacturing. Unlike the previous models, we will assume that production is followed by demand realization over time (the previous models assumed that the whole demand may occur at a time). This provides an opportunity for marketing to superficially increase realized demand when it knows that manufacturing does not have enough products to satisfy a given demand. For example, suppose marketing provided a forecast $d$ and manufacturing produced a quantity $q_{m}<d$. To reduce their own over-estimate penalty (if the penalty scheme is as in the basic model) or to make manufacturing produce more the next time, marketing might superficially provide a higher request for units than the true demand realization $D$ knowing that manufacturing can not produce it (thereby pretending that the actual demand realized is at least as large as $d$ ). We assume that there is no separate storage place reserved for the marketing department's usage after getting the supply from manufacturing so that marketing asks for more items than the realized demand only when it is aware that the amount can not be provided by manufacturing. In this model, the penalty scheme for marketing is the same as in model variant 2; however, the manufacturing department's penalty is given by $p_{u} \max \{d-q, 0\} I\{D>q\}$. Manufacturing department's objective for the new model is

$$
\min \left\{\phi_{m}^{(3)}\left(q_{m}\right), q_{m} \geq 0\right\}
$$

where

$$
\begin{aligned}
\phi_{m}^{(3)}\left(q_{m}\right)= & p_{u} E\left[\max \left\{d-q_{m}, 0\right\} I\left\{D>q_{m}\right\}\right]+c q_{m} \\
& +s E\left[\max \left\{q_{m}-D, 0\right\}\right] .
\end{aligned}
$$

We will next see that this situation will force manufacturing to produce more than that in the basic scenario.

Proposition 4. All other factors constant, the optimal manufacturing quantity for this model is greater than the optimal manufacturing quantity for the basic model (derived in Proposition 1).

Proof. To show that the optimal values for the basic model and this model are ordered, we will show that the first derivative of $\phi_{m}\left(q_{m}\right)$, the manufacturing cost for the basic model (see Equation (4)), is always greater than or equal to the first derivative of $\phi_{m}^{(3)}\left(q_{m}\right)$, the manufacturing cost for this model (see Equation (24)). Thus the ordering of the optimal production quantities.

$$
\begin{aligned}
\frac{\partial}{\partial q_{m}} & \left(\phi_{m}\left(q_{m}\right)-\phi_{m}^{(3)}\left(q_{m}\right)\right) \\
= & \frac{\partial}{\partial q_{m}}\left(p _ { u } \left[E\left[\max \left\{\min \{d, D\}-q_{m}, 0\right\}\right]\right.\right. \\
& \left.\left.-E\left[\left(\max \left\{d-q_{m}, 0\right\}\right) I\left\{D>q_{m}\right\}\right]\right]\right) \\
= & p_{u}\left(\max \left\{d-q_{m}, 0\right\}\right) f\left(q_{m}\right) \geq 0 .
\end{aligned}
$$


It is now routinely verified that the optimal order quantities in these cases are ordered.

For $q_{m}<D$, manufacturing is penalized more than the basic model because for higher demand forecasts the manufacturing department will be asked to provide the forecasted quantity by marketing. As a result, with a higher production quantity manufacturing is able to avoid some of the under-supply penalty. In addition, when the production quantity is higher, manufacturing gets to know the exact demand (which is fudged by marketing when $q_{m}<D$ ). Thus, the higher production quantity could be also be viewed as a mechanism for obtaining more information about the actual demand.

Observe that $E\left[\max \left\{d-q_{m}, 0\right\} I\left\{D>q_{m}\right\}\right]=$ $\max \left\{d-q_{m}, 0\right\}\left(1-F\left(q_{m}\right)\right)$. If $F\left(q_{m}\right)$ is concave in $q_{m}$ (that is, $f\left(q_{m}\right)$ is decreasing in $\left.q_{m}\right)$, then $E\left[\max \left\{d-q_{m}, 0\right\}\right.$ $\left.I\left\{D>q_{m}\right\}\right]$ is decreasing and convex in $q_{m}$. Then from Equation (24) we can conclude that if $f\left(q_{m}\right)$ is decreasing in $q_{m}$, then $\phi_{m}^{(3)}\left(q_{m}\right)$ is convex in $q_{m}$. Otherwise it need not be convex in $q_{m}$. In any event, a detailed analysis of the manufacturing's objective function for this model (e.g., see the proof of Proposition 1) will reveal the following result.

Proposition 5. The optimal quantity $q_{m}^{*}(d)$ produced by manufacturing for a demand forecast d made by marketing, is given by

$$
q_{m}^{*}(d)=d, \quad d \leq F^{-1}\left(\frac{p_{u}-c}{p_{u}+s}\right)
$$

and

$$
q_{m}^{*}(d)=\hat{q}_{m}<d, \quad d>F^{-1}\left(\frac{p_{u}-c}{p_{u}+s}\right),
$$

where $\hat{q}_{m}$ satisfies

$$
F\left(\hat{q}_{m}\right)=\frac{p_{u}-c}{p_{u}+s}+\frac{p_{u}}{p_{u}+s}\left(d-\hat{q}_{m}\right) f\left(\hat{q}_{m}\right) .
$$

If the density function $f$ of the demand is decreasing, then $\hat{q}_{m}$ will be the non-negative solution to Equation (27). Since manufacturing will tend to overproduce in this case, we may use marketing to coordinate the production so that a global optimality is achieved.

Theorem 5. If $p_{u} \geq p_{s}$ and $p_{o}=p_{s}(c+s) /\left(p_{s}-c\right)$, then the optimal quantities produced and sold in both centralized and decentralized systems are identical. Specifically $d^{*}=q_{m}^{*}\left(d^{*}\right)=q_{t}^{*}$.

Remark 9. Even in this model variant, we can coordinate marketing and manufacturing to perform in a globally optimal way by providing the right penalty for marketing and by providing a large enough penalty for manufacturing. Hence a proper control over marketing is essential in this case as well, since the coordination is entirely carried out through marketing (that is we need to directly control them to choose the right forecast).

\section{Models with effort}

In many situations demand for a product can be affected to a great extent by the amount of effort expended by marketing mainly through two types of activities. One set of activities does not have a tangible cost for the firm but does have a cost for the marketing department (like the marketing representatives with a fixed salary working overtime). The other set of activities has direct costs for the firm and the marketing department (like promotions, advertisements). To make our analysis tractable, we assume that all efforts in the two categories above can be captured by a single effort parameter $e$. We represent the tangible costs by $u(e)$ and the intangible costs by $v(e)$. Note that by using single effort level the mix of these two types of costs is fixed. In this section, we discuss two models where the effort $e$ expended by marketing is linked to the demand distribution in a stochastic sense. In these models, the marketing department not only determines the demand forecast but also determines the effort level to expend. The timing is the same as the models with fixed demand distribution. However, the effort level is inherent to marketing. Therefore, the true demand distribution is only known by marketing (this requires a coordination mechanism as in Model Variant 1). Here are some additional notations.

$$
\begin{aligned}
e= & \text { marketing's effort level; } \\
D(e)= & \text { demand for an effort level } e \\
F(\cdot, e)= & \text { cumulative distribution function of } \\
& \text { demand for an effort level } e \\
F^{-1}(\cdot, e)= & \text { inverse of } F(\cdot, e) ; \\
v(e)= & \text { intangible costs associated with an effort } \\
& \text { level } e ; \\
u(e)= & \text { tangible (monetary) costs associated } \\
& \text { with an effort level } e \\
F_{c}= & \text { cumulative distribution function passed } \\
& \text { by the corporate management to } \\
& \text { manufacturing in a decentralized system; } \\
\phi_{m r}(d, e)= & \text { marketing's objective for a given effort } \\
& \text { level } e \text { and forecast } d ; \\
\phi_{t}\left(q_{t}, e\right)= & \text { joint objective for a given effort level } e \\
& \text { and production } q ; \\
\phi_{c}(q, e)= & \text { actual corporate profits for a given } \\
& \text { production quantity } q \text { and an effort level } \\
& e \text { (note that this function does not } \\
& \text { include } v(e)) ;
\end{aligned}
$$

\subsection{Model with only intangible cost for marketing effort}

In this model, the effort expended by marketing does not impose any costs for the corporate (called intangible costs). This is the case when the cost of extra effort is not subsidized by the corporate management but it is inherent to the marketing department. An example of such costs would be unpaid overtime for additional effort spent by marketing. However, when marketing makes the decision 
about the effort level it needs to consider these costs. Similarly, in a centralized case, although these costs do not directly affect the expected profits, they have to be taken into consideration as hidden costs because both marketing and manufacturing are involved together in the decision process. Such cost modeling is prevalent in accounting literature where researchers have used them to model effort levels that are expended by different departments within a firm [18]. In order to have a consistent comparison between a centralized and decentralized system we assume that the cost for a given effort $v(e)$ is the same for both systems.

In this model, the penalty scheme for marketing's overestimate and manufacturing's under-supply is the same as in our basic model (described in Section 3.1). Marketing's objective is to maximize the revenues from sales less the overestimate penalty and the cost $v(e)$ of effort. We will assume that the intangible cost for effort $v(e)$ is increasing and convex in $e$. Throughout this paper we will assume that the demand is stochastically increasing in marketing's effort $e$, i.e., $\partial F(x, e) / \partial e \leq 0$ as is discussed by Shaked and Shanthikumar [19].

In a centralized system, the effort level $\left(e_{t}\right)$ and the production quantity $\left(q_{t}\right)$ are determined so as to maximize the profit after sales, production and salvage costs and the cost associated with effort. The objective is,

$$
\max \left\{\phi_{t}\left(q_{t}, e_{t}\right): q_{t} \geq 0, e_{t} \geq 0\right\},
$$

where

$$
\begin{aligned}
\phi_{t}\left(q_{t}, e_{t}\right)= & p_{s} E\left[\min \left\{q_{t}, D\left(e_{t}\right)\right\}\right]-s E\left[\max \left\{q_{t}-D\left(e_{t}\right), 0\right\}\right] \\
& -c q_{t}-v\left(e_{t}\right) .
\end{aligned}
$$

Rewriting, we get

$$
\phi_{t}\left(q_{t}, e_{t}\right)=\left(p_{s}-c\right) q_{t}-\left(p_{s}+s\right) \int_{0}^{q_{t}} F\left(x, e_{t}\right) \mathrm{d} x-v\left(e_{t}\right) .
$$

From Proposition 2 we see that for a given effort level $e_{t}$, the optimal production quantity for this case is

$$
q_{t}^{*}\left(e_{t}\right)=F^{-1}\left(\frac{p_{s}-c}{p_{s}+s}, e_{t}\right) .
$$

Note that for a fixed effort level $e_{t}$, the intangible cost $v\left(e_{t}\right)$ does not affect the optimal production quantity

$$
q_{t}^{*}\left(e_{t}\right)=F^{-1}\left(\frac{p_{s}-c}{p_{s}+s}, e_{t}\right) .
$$

The corresponding profit function for a given effort level $e_{t}$ is

$$
\begin{aligned}
\hat{\phi}_{t}\left(e_{t}\right) & =\phi_{t}\left(q_{t}^{*}\left(e_{t}\right), e_{t}\right) \\
& =\left(p_{s}-c\right) q_{t}^{*}\left(e_{t}\right)-\left(p_{s}+s\right) \int_{0}^{q_{t}^{*}\left(e_{t}\right)} F\left(x, e_{t}\right) \mathrm{d} x-v\left(e_{t}\right) .
\end{aligned}
$$

Since the cost associated with marketing's effort does not affect the corporate profit (in a direct way) in this model, the corporate profit for a marketing's effort level $e$ and manufacturing's production quantity $q$ is given by,

$$
\begin{aligned}
\phi_{c}(q, e)= & p_{s} E[\min \{q, D(e)\}] \\
& -s E[\max \{q-D(e), 0\}]-c q .
\end{aligned}
$$

(Compare this to Equation (15)). For a fixed effort level the optimal corporate production quantity is given by (see Proposition 2),

$$
q_{c}^{*}(e)=F^{-1}\left(\frac{p_{s}-c}{p_{s}+s}, e\right)=q_{t}^{*}(e) .
$$

Since $F(x, e)$ is increasing in $x$ and decreasing in $e$ (and hence $F^{-1}(y, e)$ is increasing in $y$ and $e$ ), we have $q_{c}^{*}(e)$ increasing in $e$. The optimal corporate profit for a fixed effort level $e$ is then given by

$$
\begin{aligned}
\hat{\phi}_{c}^{*}(e)= & \phi_{c}\left(q_{c}^{*}(e), e\right)=p_{s} E\left[\min \left\{q_{c}^{*}(e), D(e)\right\}\right] \\
& -s E\left[\max \left\{q_{c}^{*}(e)-D(e), 0\right\}\right]-c q_{c}^{*}(e) .
\end{aligned}
$$

In a decentralized system, for a given effort level, marketing's objective is,

$$
\max \left\{\phi_{m r}(d, e), d \geq 0, e \geq 0\right\},
$$

where

$$
\begin{aligned}
\phi_{m r}(d, e)= & p_{s} E\left[\min \left\{D(e), q_{m}^{*}(d)\right\}\right] \\
& -p_{o} E[\max \{d-D(e), 0\}]-v(e),
\end{aligned}
$$

where, $q_{m}^{*}(d)$ is the quantity produced by manufacturing in response to marketing's forecast of $d$. Rewriting the above objective we have,

$$
\begin{aligned}
\phi_{m r}(d, e)= & p_{s} q_{m}^{*}(d)-p_{s} \int_{0}^{q_{m}^{*}(d)} F(x, e) \mathrm{d} x \\
& -p_{o} \int_{0}^{d} F(x, e) \mathrm{d} x-v(e) .
\end{aligned}
$$

Marketing's effort level cannot be observed by the corporate management and by the manufacturing department, because the effort level is inherent to marketing and they may not reveal it. As a result, in a decentralized case, it is important for the corporate management to pass an appropriate demand distribution to manufacturing in order to maintain the coordination between forecast and production quantities. As shown in Section 3.2, it is possible to set incentives and for the corporate management to pass a distribution to manufacturing so that it always produces what marketing asks for. We assume that such an arrangement exists in this and the next model (that is, we have, $q_{m}^{*}(d)=d$ ). Then for a given effort level we have (see Proposition 2)

$$
q_{m}^{*}\left(d^{*}(e)\right)=d^{*}(e)=F^{-1}\left(\frac{p_{o}}{p_{o}+p_{s}}, e\right) .
$$


Now, from Equations (37) and (38) one sees that the marketing's optimal revenue for a given effort level $e$ is given by

$$
\begin{aligned}
\hat{\phi}_{m r}(e) & =\phi_{m r}\left(d^{*}(e), e\right) \\
& =p_{s} d^{*}(e)-\left(p_{s}+p_{o}\right) \int_{0}^{d^{*}(e)} F(x, e) \mathrm{d} x-v(e) .
\end{aligned}
$$

It is to be noted that the effort levels to be spent are determined using different objectives in centralized and decentralized systems. In the decentralized system, marketing sets the effort level to be expended by maximizing its own profit. In the centralized system, however, the optimum effort level is determined by marketing and manufacturing together using the joint objective.

Proposition 6. If demand is stochastically increasing in effort (that is, $\partial F(x, e) / \partial e \leq 0)$, and the over-estimate penalty $p_{o}$ for marketing is such that $s \leq p_{o} \leq$ $p_{s}(s+c) /\left(p_{s}-c\right)$ then marketing will put more effort in a decentralized system than the effort in a centralized system.

Proof. We will show the above result by comparing the partial derivatives for both the marketing and joint objectives with respect to effort $e$. From Equations (32) and (39), we get,

$$
\begin{gathered}
\frac{\partial \hat{\phi}_{m r}(e)}{\partial e}=p_{s} \frac{\partial}{\partial e} d^{*}(e)-\left(p_{s}+p_{o}\right) \int_{0}^{d^{*}(e)} \frac{\partial}{\partial e} F(x, e) \mathrm{d} x \\
-\left(p_{s}+p_{o}\right) F\left(d^{*}(e), e\right) \frac{\partial}{\partial e} d^{*}(e)-v^{\prime}(e), \\
\frac{\partial \hat{\phi}_{t}(e)}{\partial e}=\left(p_{s}-c\right) \frac{\partial}{\partial e} q_{t}^{*}(e)-\left(p_{s}+s\right) \int_{0}^{q_{t}^{*}(e)} \frac{\partial}{\partial e} F(x, e) \mathrm{d} x \\
-\left(p_{s}+s\right) F\left(q_{t}^{*}(e), e\right) \frac{\partial}{\partial e} q_{t}^{*}(e)-v^{\prime}(e),
\end{gathered}
$$

where

$$
v^{\prime}(e)=\frac{\partial}{\partial e} v(e)
$$

$\hat{\phi}_{m r}(e)$ attains its maximum at a higher effort level $e$ than $\hat{\phi}_{t}(e)$ if

$$
\frac{\partial}{\partial e}\left(\hat{\phi}_{m r}(e)-\hat{\phi}_{t}(e)\right) \geq 0
$$

That is, if

$$
-\left(p_{s}+p_{o}\right) \int_{0}^{d^{*}(e)} \frac{\partial}{\partial e} F(x, e) \mathrm{d} x+\left(p_{s}+s\right) \int_{0}^{q_{t}^{*}(e)} \frac{\partial}{\partial e} F(x, e) \mathrm{d} x \geq 0,
$$

we get the above expression because $F\left(d^{*}(e), e\right)=$ $p_{s} /\left(p_{o}+p_{s}\right)$ and $F\left(q_{t}^{*}(e), e\right)=\left(p_{s}-c\right) /\left(p_{s}+s\right)$ for a given effort $e$. Since $p_{o} \leq p_{s}(s+c) /\left(p_{s}-c\right)$ implies that $d^{*}(e) \geq q_{t}^{*}(e)$. We know that $\partial F(x, e) / \partial e \leq 0$ and $p_{o} \geq s$. Thus, the above inequality is valid, implying that the effort applied by marketing is greater in a decentralized system than in a centralized system.

Now we want to consider the expected profits realized for the firm in the decentralized and the centralized system.

Proposition 7. The expected profits for the firm can be higher in the decentralized system if $p_{o}=p_{s}(s+c)$ / $\left(p_{s}-c\right)$.

Proof. We know from Theorem 4 that if $p_{o}=p_{s}(s+c) /$ $\left(p_{s}-c\right)$ and $p_{u} \geq p_{s}$ then for a given effort level $e=e_{t}$, the expected profits in the centralized and decentralized systems are equal. For any given effort level, we can always equate the quantity produced in both cases by setting the penalty scheme in the above manner. Now, the real corporate optimal profit function for a fixed effort level $e$ is given by (see Equation (34))

$$
\begin{aligned}
\hat{\phi}_{c}(e)= & \left(p_{s}-c\right) q_{c}^{*}(e)-\left(p_{s}+s\right) \int_{0}^{q_{c}^{*}(e)} x f(x, e) \mathrm{d} x \\
& -\left(p_{s}+s\right) F\left(q_{c}^{*}(e), e\right) .
\end{aligned}
$$

The derivative of the corporate profit with respect to effort $e$ is given by

$$
\begin{aligned}
\frac{\partial \hat{\phi}_{c}(e)}{\partial e}= & \left(p_{s}-c\right) \frac{\partial}{\partial e} q_{c}^{*}(e)-\left(p_{s}+s\right) \int_{0}^{q_{c}^{*}(e)} \frac{\partial}{\partial e} F(x, e) \mathrm{d} x \\
& -\left(p_{s}+s\right) F\left(q_{c}^{*}(e), e\right) \frac{\partial}{\partial e} q_{c}^{*}(e) .
\end{aligned}
$$

Since we know that, $F\left(q_{c}^{*}(e), e\right)=\left(p_{s}-c\right) /\left(p_{s}+s\right)$, we get

$$
\frac{\partial \hat{\phi}_{c}(e)}{\partial e}=-\left(p_{s}+s\right) \int_{0}^{q_{c}^{*}(e)} \frac{\partial}{\partial e} F(x, e) \mathrm{d} x .
$$

Also, we know that $\partial F(x, e) / \partial e \leq 0$, thus $\partial \hat{\phi}_{c}(e) / \partial e \geq 0$. For any given effort level, $q_{t}^{*}(e)=q_{m}^{*}(e)=d^{*}(e)$, since $p_{o}=p_{s}(s+c) /\left(p_{s}-c\right)$. Thus, for any given effort level the performance of decentralized and centralized systems are identical and in addition we know that the real corporate profit function is increasing in the effort level. From Proposition 6, we know that effort in a decentralized system is greater than or equal to the effort in a centralized system. Therefore, the expected profits in the centralized system is smaller than the expected profits in the decentralized system. 


\subsection{Model with tangible and intangible costs for marketing effort}

In this section we consider a model where a part of the cost of the effort expended by marketing affects the corporate objective function (called tangible cost) such as the cost of advertisements and promotions. The manufacturing department decides on the optimum quantity based on its own costs and the demand distribution that it obtains from the corporate management. Let $u(e)$ be the tangible cost and $v(e)$ be the intangible cost for an effort level $e$ both of which are increasing and convex in the effort $e$. In the decentralized case, we still assume that the corporate management provides manufacturing with a demand distribution so that marketing always gets what it asks for. Marketing's objective is,

$$
\max \left\{\phi_{m r}(d, e): d \geq 0, e \geq 0\right\},
$$

where

$$
\begin{aligned}
\phi_{m r}(d, e)= & p_{s} E\left[\min \left\{D, q_{m}^{*}(d)\right\}\right]-p_{o} E[\max \{d-D, 0\}] \\
& -u(e)-v(e) .
\end{aligned}
$$

As before assuming that we have set up a penalty scheme and a distribution $\hat{F}$ for manufacturing so that $q_{m}^{*}(d)=d$ we have that the optimal revenue for marketing for a given effort level $e$ is given by (compare this to Equation (39)),

$$
\begin{aligned}
\hat{\phi}_{m r}(e)= & \phi_{m r}\left(d^{*}(e), e\right)=p_{s} d^{*}(e)-\left(p_{s}+p_{o}\right) \\
& \times \int_{0}^{d^{*}(e)} F(x, e) \mathrm{d} x-v(e)-u(e) .
\end{aligned}
$$

In the centralized case, both the tangible and intangible costs of effort are taken into consideration. The joint objective is,

$$
\max \left\{\phi_{t}\left(q_{t}, e_{t}\right): q_{t} \geq 0, e_{t} \geq 0\right\},
$$

where

$$
\begin{aligned}
\phi_{t}\left(q_{t}, e_{t}\right)= & p_{s} E\left[\min \left\{q_{t}, D\left(e_{t}\right)\right\}\right]-s E\left[\max \left\{q_{t}-D\left(e_{t}\right), 0\right\}\right] \\
& -c q_{t}-u\left(e_{t}\right)-v\left(e_{t}\right) .
\end{aligned}
$$

As before (see Equation (32)), the optimal profit for joint decision making with a fixed effort level $e_{t}$ is

$$
\begin{aligned}
\hat{\phi}_{t}\left(e_{t}\right)= & \phi_{t}\left(q_{t}^{*}\left(e_{t}\right), e_{t}\right)=\left(p_{s}-c\right) q_{t}^{*}\left(e_{t}\right)-\left(p_{s}+s\right) \\
& \times \int_{0}^{q_{t}^{*}\left(e_{t}\right)} F\left(x, e_{t}\right) \mathrm{d} x-u\left(e_{t}\right)-v\left(e_{t}\right) .
\end{aligned}
$$

Proposition 8. If demand is stochastically increasing in effort and overestimate penalty for marketing is set such that $s \leq p_{o} \leq p_{s}(s+c) /\left(p_{s}-c\right)$, marketing will put more effort in a decentralized system than the optimal level for a centralized system.
Proof. The above result can be proved with similar arguments as in the proof of Proposition 6.

The corporate's profit for marketing's effort level $e$ and manufacturing's production quantity $q$ is given by,

$$
\begin{aligned}
\phi_{c}(q, e)= & p_{s} E[\min \{q, D(e)\}]-s E[\max \{q-D(e), 0\}] \\
& -c q-u(e) .
\end{aligned}
$$

(Compare this to Equations (15) and (33)). The corporate profit function $\phi_{c}(q, e)$ is no longer monotone in effort $e$ because there is a tangible cost $u(e)$ which is included in the objective function. However, for a fixed effort level the optimal corporate production quantity is still given by (see Proposition 2),

$$
q_{c}^{*}(e)=F^{-1}\left(\frac{p_{s}-c}{p_{s}+s}, e\right) .
$$

Since $F(x, e)$ is increasing in $x$ and decreasing in $e$ (and hence $F^{-1}(y, e)$ is increasing in $y$ and $e$ ), we have $q_{c}^{*}(e)$ increasing in $e$. The optimal corporate profit for a fixed effort level $e$ is then given by

$$
\begin{aligned}
\hat{\phi}_{c}^{*}(e)= & \phi_{c}\left(q_{c}^{*}(e), e\right)=p_{s} E\left[\min \left\{q_{c}^{*}(e), D(e)\right\}\right] \\
& -s E\left[\max \left\{q_{c}^{*}(e)-D(e), 0\right\}\right]-c q_{c}^{*}(e)-u(e) .
\end{aligned}
$$

Rewriting this we get,

$$
\hat{\phi}_{c}(e)=\left(p_{s}-c\right) q_{c}^{*}(e)-\left(p_{s}+s\right) \int_{0}^{q_{c}^{*}(e)} F(x, e) \mathrm{d} x-u(e) .
$$

The optimal effort level for maximum corporate profit is $e^{*}$ and is obtained by maximizing $\hat{\phi}_{c}(e)$.

We know from Proposition 8, that marketing expends more effort (say, $e_{m r}^{*}$ ) in the decentralized system than the effort (say, $e_{t}^{*}$ ) in a centralized system. Since an additional cost $v(e)$ increasing in the effort level $e$ is involved while computing $e_{t}^{*}$ we know that $e_{t}^{*} \leq e^{*}$ (Compare Equations (45) and (48)). In addition, since $\hat{\phi}(e)$ is not necessarily increasing in $e$, it is possible that marketing expends more effort than is globally optimal and may incur more tangible costs (that is, for $e_{m r}^{*}>e_{t}^{*}$ it is possible to have $\left.\hat{\phi}_{c}\left(e_{m r}^{*}\right)<\hat{\phi}_{c}\left(e_{t}^{*}\right)<\hat{\phi}_{c}\left(e^{*}\right)\right)$. In order to avoid this, the corporate management needs to develop a mechanism so that the effort spent in the decentralized case is such that the corporate profit is still larger than that in the centralized case (that is, $\left.\hat{\phi}_{c}\left(e^{*}\right) \geq \hat{\phi}_{c}\left(e_{m r}^{*}\right) \geq \hat{\phi}_{c}\left(e_{t}^{*}\right)\right)$. Next we will discuss such a possibility.

Suppose $\hat{\phi}_{c}(e)$ is concave in $e$ and let $e^{*}$ be the optimal effort level that maximizes $\hat{\phi}_{c}(e)$. Then $\hat{\phi}_{c}(e)$ is increasing in $e\left(e \leq e^{*}\right)$ and decreasing in $e\left(e \geq e^{*}\right)$. Hence, if we can guarantee that marketing's effort will not exceed $e^{*}$, then we can guarantee that $\hat{\phi}_{c}\left(e_{m r}^{*}\right) \geq \hat{\phi}_{c}\left(e_{t}^{*}\right)$. The corporate management can achieve this by setting a budget constraint $B=u\left(e^{*}\right)$ for the tangible costs of marketing. With this constraint, a larger corporate profit is obtained with 
a larger effort. We will next present a sufficient condition under which this can be achieved.

Proposition 9. Suppose (i) the corporate management sets a marketing budget $B$ for tangible costs associated with marketing efforts such that $B=u\left(e^{*}\right)$ where $e^{*}$ is the effort level that maximizes the corporate profit, (ii) $p_{u} \geq p_{s}$, (iii) $p_{o}=p_{s}(s+c) /\left(p_{s}-c\right)$, (iv) manufacturing is given a demand distribution $F_{c}(x) \leq F\left(x, e^{*}\right), x \geq 0$ and $(\mathrm{v}) F(x, e)$ is decreasing in e and $\int_{0}^{y} F(x, e) \mathrm{d} x$ is jointly convex in $(y, e)$. Then the profits from a decentralized system will still be greater than or equal to the profits from a centralized system.

Proof. First we will show that $\hat{\phi}_{c}(e)$ is concave in $e$. For this consider,

$$
\begin{aligned}
\phi_{c}(q, e)= & p_{s} E[\min \{q, D(e)\}]-s E[\max \{q-D(e), 0\}] \\
& -c q-u(e) .
\end{aligned}
$$

Rewriting it we get

$$
\phi_{c}(q, e)=\left(p_{s}-c\right) q-\left(p_{s}+s\right) \int_{x=0}^{q} F(x, e) \mathrm{d} x-u(e) .
$$

Since $\int_{x=0}^{q} F(x, e) \mathrm{d} x$ is jointly convex in $(q, e)$, and $u(e)$ is convex in $e$, it is immediately clear that $\phi_{c}(q, e)$ is jointly concave in $(q, e)$. Hence $\hat{\phi}_{c}(e)$ is concave in $e$. Next the conditions (ii) $p_{u} \geq p_{s}$, (iii) $p_{o}=p_{s}(s+c) /\left(p_{s}-c\right)$, and (iv) manufacturing is given a demand distribution $F_{c}(x) \leq F\left(x, e^{*}\right), x \geq 0$ guarantees that marketing's demand forecast $d^{*}(e)$ and manufacturing's production quantity $q_{m}^{*}\left(d^{*}(e)\right)$ are matched (see Theorem 4). Now let $e_{m r}^{*}$ be the optimal effort determined by marketing and $e_{t}^{*}$ be the optimal effort determined in a centralized system. From Proposition 8, we know that $e_{m r}^{*} \geq e_{t}^{*}$. The budget constraint does not affect this result because $u\left(e_{t}^{*}\right) \leq u\left(e^{*}\right)$ since $e_{t}^{*} \leq e^{*}$. In the presence of the budget constraint, $e_{m r}^{*} \leq e^{*}$, thus, the effort in the decentralized system is forced to be less than or equal to the effort level that maximizes the corporate profit. So, $e_{t}^{*} \leq e_{m r}^{*} \leq e^{*}$. Since the optimal corporate profit function for a given effort level $e$ is concave in $e$, the expected profit for the firm in a decentralized system is greater than or equal to that in a centralized system.

\section{Conclusions}

In this paper, we study coordination mechanisms through penalty schemes between manufacturing and marketing departments which enable organizations to match marketing efforts with production quantities. We consider two possible organizational structures - centralized and decentralized. In the decentralized case we model a single period problem as a Stackelberg game. The demand is uncertain and the marketing department, the leader, provides a forecast to manufacturing, the follower, which in turn produces, based on the forecast and the demand distribution. In the centralized model, marketing and manufacturing jointly decide on the production quantity. Among other results we show that by setting suitable penalties one can generate the same result in a decentralized system as that obtained from a centralized system. We also show that setting the correct penalty for marketing is essential for coordination. We also analyze models where the marketing department has the ability to change the distribution of demand based on efforts (through promotion, advertising and personal relationships with customers). An interesting result indicates that it is possible to set penalties so that a coordinated decentralized system outperforms a centralized system when there are no tangible costs to the organization for the efforts expended by the marketing department.

In this paper, we have tried to understand the coordination issues between marketing and manufacturing departments using simple models. The models studied in this paper provide insights on some of the important issues related to coordination between marketing and manufacturing departments. The main restrictions in our models are as follows. Firstly, we restricted ourselves to a single period analysis which is suitable for products with a very short life cycle. For multi-period problems where a myopic policy is efficient, the results from this model can be usefully applied. Secondly, there are several other factors that affect the coordination including manufacturing capacity, information systems capabilities and organizational issues which we have neglected in our models. We feel that incorporating these additional factors will complicate the model and make it difficult to analyze. Finally, we restricted our attention to a single channel for marketing. In many real situations, there could be multiple channels and the demands generated are often correlated. Under those conditions, it will become difficult to coordinate and this is a topic for future research.

\section{References}

[1] Eliashberg, J. and Steinberg, R. (1993) Marketing-production joint decision-making, in Handbooks in Operations Research and Management Science, Vol. 5, Eliashberg, J. and Lilien, G.L. (eds), Elsevier, Amsterdam, The Netherlands, pp. 827-880.

[2] Fudenberg, D. and Tirole, J. (1991) Game Theory, MIT Press, Cambridge, MA.

[3] Porteus, E.L. and Whang, S. (1991) On manufacturing/marketing incentives. Management Science, 37(9), 1166-1181.

[4] De Groote, X. (1991) Stochastic inventory systems in a supply chain with asymmetric information, flexibility and marketing/ manufacturing coordination. Working Paper, INSEAD, Fontainbleau, France.

[5] Hartman, B. and Dror, M. (1996) Cost allocation in continuousreview inventory models. Naval Research Logistics, 43, 549-561.

[6] Cachon, G. and Lariviere, M. (1997) Contracting to assure supply or what did the supplier know and when did he know it? Working Paper, Fuqua School of Business, Duke University, Durham, NC. 
[7] Corbett, C.J. (1997) Stochastic inventory systems in a supply chain with asymmetric information. Working Paper, Anderson School of Management, UCLA, Los Angeles, CA.

[8] Shapiro, B.P. (1977) Can marketing and manufacturing coexist. Harvard Business Review, 55(5), 104-114.

[9] Crittenden, V.L., Gardiner, L.R. and Stam, A. (1993) Reducing conflict between marketing and manufacturing. Industrial Marketing Management, 22(4), 299-309.

[10] Kahn, K.B. and Mentzer, J.T. (1994) Norms that distinguish between marketing and manufacturing. Journal of Business Research, 30(2), 111-118.

[11] St. John, C.H. and Hall, E.H. Jr. (1991) The interdependency between marketing and manufacturing. Industrial Marketing Management, 20(3), 223-229.

[12] Lee, W.J. and Kim, D.S. (1993) Optimal and heuristic decision strategies for integrated production and marketing planning. Decision Sciences, 24(6), 1203-1213.

[13] Kumar, K.R. and Hadjinicola, G.C. (1996) Resource allocation to defensive marketing and manufacturing strategies. European Journal of Operational Research, 94(3), 453-466.

[14] Leitch, R.A. (1974) Marketing strategy and the optimal production schedule. Management Science, 21(3), 302-312.

[15] Abad, P.L. (1987) A hierarchical optimal control model for coordination of functional decisions in a firm. European Journal of Operational Research, 32, 62-75.

[16] Damon, W.W. and Schramm, R. (1972) A simultaneous decision model for production, marketing and finance. Management Science, 19(2), 161-172.

[17] Freeland, J.R. (1980) Coordination strategies for production and marketing in a functionally decentralized firm. AIIE Transactions, 12(2), 126-132.

[18] Baiman, S. (1990) Agency theory in managerial accounting: a second look. Accounting Organizations and Society, 15(4), 341371.

[19] Shaked, M. and Shantikumar, J.G. (1994) Stochastic Orders and Their Applications, Academic Press, San Diego, California.

\section{Biographies}

Muruvvet Celikbas received her B.S. degrees in Mechanical Engineering and Industrial Engineering from Bogazici University in Istanbul,
Turkey. She holds an M.S. degree in Industrial Engineering and Operations Research from the University of California, Berkeley. She is currently a Ph.D. student at the Industrial Engineering and Operations Research Department at University of California, Berkeley. Her research interests are in stochastic models of manufacturing systems, marketing/manufacturing interface and coordination issues in supply chains.

J. George Shanthikumar received a B.Sc. degree in Mechanical Engineering from the University of Sri Lanka in 1972 and the M.A.Sc. and $\mathrm{Ph} . \mathrm{D}$. degrees in Industrial Engineering from the University of Toronto, Ontario, Canada, in 1977 and 1979, respectively. He is Professor of Industrial Engineering and Operations Research (in the College of Engineering) and Manufacturing and Information Technology (in the Walter A. Haas School of Business) at the University of California, Berkeley. His research interests are in production and service systems modeling and analysis, queueing theory, reliability, scheduling, stochastic processes, simulation and supply chain management. He has written or written jointly over 250 technical papers on these topics. $\mathrm{He}$ is a coauthor (with John A. Buzacott) of the book Stochastic Models of Manufacturing Systems and a coauthor (with Moshe Shaked) of the book Stochastic Orders and Their Applications. He is (or was) a member of the editorial boards of the IIE Transactions on Design and Manufacturing, International Journal of Flexible Manufacturing Systems, Journal of Discrete Event Dynamic Systems, Journal of Production and Operations Management, Operations Research, Operations Research Letters, OPSEARCH, Probability in the Engineering and Informational Sciences, and Queueing Systems: Theory and Applications.

Jayashankar M. Swaminathan is an Assistant Professor in the area of Manufacturing and Information Technology at the Walter A. Haas School of Business, University of California, Berkeley. He obtained his M.S. and Ph.D. degrees in Industrial Administration from Carnegie Mellon University, Pittsburgh and his B.Tech degree in Computer Science and Engineering from the Indian Institute of Technology, Delhi. His research interests are in the areas of supply chain management, product variety and stochastic manufacturing systems. The research in his dissertation was awarded the George E. Nicholson Prize (best student paper) and an honorable mention in the George B. Dantzig Prize (dissertation prize) by the Institute for Operations Research and the Management Sciences (INFORMS). His research is motivated by interaction with firms such as IBM, 3Com, Sun Microsystems and Ford. 